\title{
Previsão de Duração de Paradas de Linha em uma Indústria Automotiva
}

\author{
Forecast of Duration of Line Stops in an Automotive Industry \\ Eduardo Henrique Soares Viana 1 (D) orcid.org/0000-0001-8116-5314 \\ Gabriel Lenon Barros da Silva ${ }^{1}$ (D) orcid.org/0000-0002-0909-5152 \\ Leonardo de Carvalho Guerra ${ }^{1}$ (D) orcid.org/0000-0001-7231-068X \\ Wendel Vanderley da Paz ${ }^{1}$ (D) orcid.org/0000-0003-0607-1750 \\ ${ }^{1}$ Escola Politécnica de Pernambuco, Universidade de Pernambuco, Recife, Brasil. \\ E-mail do autor principal: Gabriel Silva glbs@ecomp.poli.br
}

\section{Resumo}

Processos de Manufatura Avançada (P.M.A.) necessitam de rigoroso controle da produção e de qualidade do produto manufaturado final. Neste cenário, perdas produtivas ocorrem, o que acarreta em aumento nos custos de produção. Portanto, é proposta uma previsão do perfil e do tipo de parada de linha, dada entrada de um evento de parada de linha que ocorre em tempo real. Utilizando a proposta de Mineração de dados dos resultados entre os anos de 2018 até 2020 do histórico de paradas, à luz da metodologia CRISP-DM, foram encontrados valores de acurária na medição do tempo de parada de linha com a abordagem do Random Forest. Em um cenário de desbalanceamento das classes da base de dados, foi encontrado $51 \%$ de acurária, enquanto que com o balanceamento das mesmas classes e repetição dos testes para valores distintos, foi atingido 38\% de acurácia. Por fim, o surgimento desse perfil de perda e de tempo a ser previsto auxiliará na tomada de decisão do gerente do negócio quando eventos de paradas de produção para reparação de equipamentos serão necessários de acontecer, para prevenir grandes paradas de linha.

Palavras-Chave: Previsão; Floresta Aleatória; Paradas de produção; Indústria Automotiva; Mineração de dados.

\section{Abstract}

Advanced Manufacturing Processes (A.M.P.) require strict production and quality control of the final manufactured product. In this scenario, productive losses occur, which leads to an increase in production costs. Therefore, a forecast of the profile and type of line stop is proposed, given the entry of a line stop event that occurs in real-time. Using the proposed data mining between the years 2018 to 2020 of the stop history, in the light of the CRISP-DM methodology, accuracy parameters were calculated in the measurement of the line stop time with the Random Forest approach. In a scenario of imbalance of the classes in the database, $51 \%$ accuracy was found, while with the balance of the same classes and repetition of tests for different values, $38 \%$ accuracy was achieved. Finally, the appearance of this profile of loss and time to be foreseen will assist in the decision making of the business manager when, events of production stoppages for equipment repair will be necessary to happen, to prevent major line stoppages.

Key-words: Forecasting; Random Forest; Production Stop Line; Automotive Industry; Data Mining 


\section{Introdução}

\subsection{Contextualização}

Processos de Manufatura Avançada (P.M.A.) necessitam de rigoroso controle da cadeia produtiva e da qualidade dos itens a serem fabricados [1]. Ferramentas de gestão de processos foram concebidas para auxiliar na análise de problemas, bem como na busca de possíveis causa-raízes e motivos que levaram a ocorrência de falha na cadeia de produção [2], [3].

Com o advento da Manufatura 4.0, sistemas informatizados que atuam em tempo real com o processo de produção, chamados de Manufacturing Execution System (M.E.S., ou Sistema de Execução da Manufatura, tradução-livre do inglês) surgiram, a fim de garantir o supervisão do fluxo fabril através de sistemas computacionais [1]-[4].

Sendo assim, ao longo do processo de manufatura automotiva, surgem uma grande quantidade de dados e registros das operações, peças e consumo de itens utilizados no processo de fabricação, recursos esses que se tratados de maneira adequada e disponibilizados para tomada de decisão do gestor do processo, podem facilitar qual estratégia de negócio e de produção deve ser tomada [4]-[8].

As ferramentas de gestão de processo possuem certas limitações com relação a análise de grande volume de dados, o que torna as tomadas de decisão restritas à análises de maneira empírica, dado experiências pontuais com os problemas e falhas que ocorrem na cadeia de produção, onde pode se considerar ou não a repetibilidade das ocorrências [9]. Em contrapartida, um aspecto positivo da utilização de sistemas integrados da Manufatura 4.0 é a possibilidade da coleta dessa informação, pois ela gera padrões que podem ser encontrados, analisados e assim serem tratados de certa forma com técnicas computacionais que tem por base a mineração dos dados [2], [4], [5], [7], [10].

\subsection{Descrição do problema}

Interrupções em processos de manufatura ocorrem por diversos motivos, e cada um possui o que é chamado de modo de falha [2], ou seja, como a falha veio a ocorrer. Em uma linha de produção contínua, esses modos de falha são motivo para registro de paradas de linha, e assim perda financeira.
Em uma linha de produção de uma indústria do setor automotivo, o processo contínuo de fabricação de veículos automotores pode sofrer de paradas de linha por registro dos colaboradores de cada estação de trabalho, por parada de ferramenta, por falta de qualidade das peças, por falta de entrega de peças no tempo correto [8].

Registros são coletados do tempo inicial, final, de qual tipo de parada são coletados, porém quando aplicadas as ferramentas de gestão de processos, muitas delas ficam restritas a análises pontuais do problema, onde se considera ou não sua recorrência, o que depende de cada caso.

\subsection{Objetivos}

\subsubsection{Objetivo Geral}

Previsão do perfil e do tipo de parada de linha (quanto tempo durarão as paradas de linha no instante em que são geradas, se causada por operador ou máquina), dada entrada de um evento de parada de linha que ocorre em tempo real.

\subsubsection{Objetivos Específicos}

- Tendência de parada de linha de acordo com as operações executadas nas estações de trabalho dos colaboradores;

- Projeção das possíveis paradas de linha que possam impactar, com maior ou menor tempo, futuras ocorrências;

- Modelo de previsão retornar valores com a maior precisão possível de estimativa de tempo para longas paradas de linha, pois assim será possível mobilizar o gestor do negócio e o gestor da manutenção nas melhores estratégias para retorno das condições de base da linha de produção.

\subsection{Justificativa}

A linha de produção da indústria a ser analisada possui capacidade produtiva de $1.040 \mathrm{carros} / \mathrm{dia}$ sendo que, por conta de paradas de linha, a mesma atinge 920 carros/dia de produção real. É de interesse do operador do negócio o estudo, dos últimos 3 anos (de 2018 até 2020), de todas as paradas de linha, a fim de que se encontre oportunidades de ganho de produção e tempo na execução das operações em todo o processo, bem como reduzir o tempo ocioso de parada de linha, ou seja, procurar entender os modo de falha, para reduzir sua ocorrência. 


\section{Fundamentação Teórica}

Para abordagem das informações que são geradas a todo momento no ambiente industrial, a metodologia CRISP-DM, que é a abreviação de Cross Industry Standard Process for Data Mining que, trazendo para o português, pode ser entendida como processo padrão da indústria cruzada para mineração de dados, foi elaborada. Essa é uma metodologia capaz de transformar os dados da empresa em conhecimento e informações de gerenciamento.

Criada na década de 1990, a CRISP-DM surgiu da necessidade dos profissionais de Data Mining de padronização do processo de análise e mineração de grande quantidade de dados. O CRISP-DM surgiu justamente para atender aos projetos que estão diretamente envolvidos com o processamento e a análise de um grande volume de dados. [11]

O DM faz parte de Data Science, que utiliza estatística e matemática como base para cruzamento de dados, por meio de técnicas de indução para propor hipóteses e solucionar questões empresariais. De maneira simplificada, é a mineração de dados que vai conseguir transformar todo o volume de dados em informações úteis para o gerenciamento e a tomada de decisões.

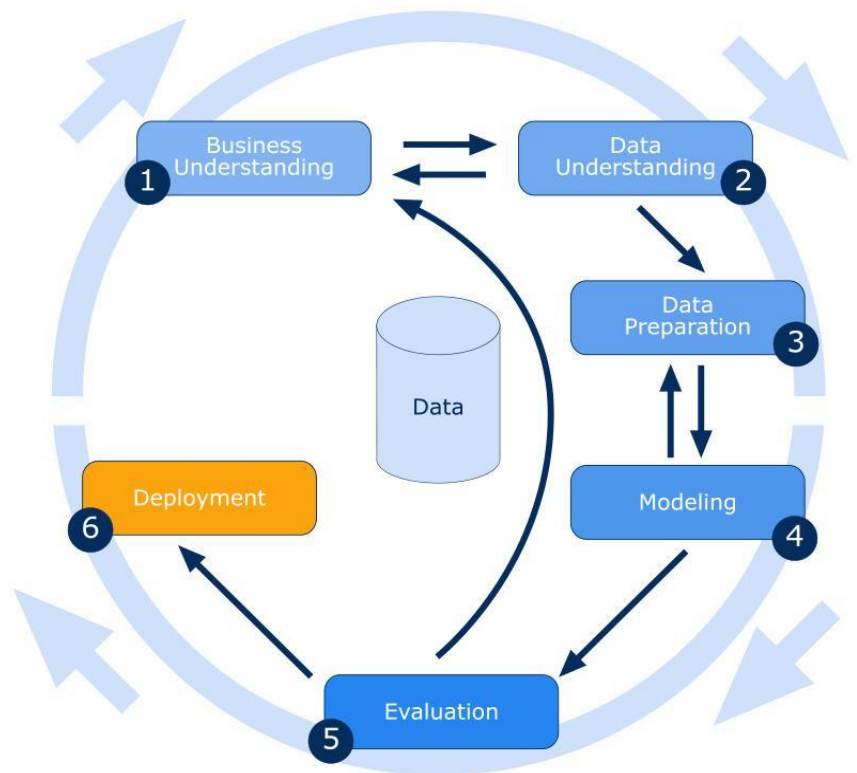

Figura 1: Ciclo de gerenciamento e implementação do CRISP-DM, e suas 6 etapas de implementação. Fonte: https://semantix.com.br/como-explorar-egerenciar-dados-com-o-crisp-dm/

\section{1 Área do Negócio}

A busca por competitividade entre grandes marcas no mercado, bem como a constante melhoria dos processos de fabricação, faz com que novos modelos e estruturas de organização da manufatura venham a surgir como resultado final desses fatores citados. Modelos de produção em grande escala, com definições de tarefas a serem executadas por mãode-obra, seja ela qualificada ou não, foram conceituados nas revoluções industriais ocorridas nos séculos XIX e XX [12].

Dos modelos de organização industrial existentes, três exemplos se destacam, dos quais: Taylorismo, Fordismo e Toyotismo. Do primeiro citado, o Taylorismo, temos a criação do conceito de Administração Científica de um processo de produção [12], que se caracteriza por:

- Priorização do métodos científicos, em face dos empíricos;

- Recrutamento do melhor colaborador para cada parte do processo de manufatura;

- Capacitação constante do colaborador;

- Cooperação entre líderes e liderados:

- $\quad$ Estudos de tempos e movimentos dos colaboradores.

Seguindo as definições, o Fordismo foi conceituado por Henry Ford como uma adaptação do Taylorismo: com a adição de um processo contínuo e ininterrupto, a exemplo uma "esteira rolante", ditando assim um novo ritmo de trabalho. Com isso, Henry Ford conseguiu reduzir ainda mais o tempo no processo de manufatura, além de permitir a introdução de maquinário trabalhando em conjunto com seres humanos, como também fazendo com que fornecedores de peças e itens a serem montados no produto ficassem localizados cada vez mais próximos do processo de montagem final [12].

Com a mudança no perfil de consumo das pessoas nos séculos XX e XXI, um sistema surgido no Japão pós-segunda guerra mundial, chamado Toyotismo, emergiu com o conceito de fabricação simples e enxuta [12]. Nesse modelo de produção, os lotes de produção são baseados na demanda dos clientes, não possuindo assim estoque como no Taylorismo e Fordismo, o que já evita desperdício de materiais e mão-de-obra. Além disso, o colaborador executa várias tarefas em escala (multitarefas) e 
existe uma integração maior com o espaço de trabalho.

Para o setor automotivo, o modelo Toyotista ganhou cada vez mais espaço, também com o advento da manufatura enxuta [13], que pelo seu conceito visa cada vez mais a melhoria do processo com o controle e diminuição dos custos e tempo de produção, mantendo a qualidade de todo o processo, em conjunto com a manufatura 4.0 , recebendo esse nome por ser contemporânea a $4^{a}$ revolução industrial - uso de tecnologias de ponta e processos computacionais [2], [14]. Sendo assim, possui como meta a integração dos processos de manufatura a sistemas computacionais de controle de produção, qualidade e tempo de operação na linha contínua. Adicionalmente, o conceito de Manufatura de Classe Mundial (do inglês World Class Manufacturing - W.C.M.) surge já na década de 1980 para integrar as diversas definições de melhoria de processo de produção da manufatura, visando sempre o controle do tempo de execução, qualidade, segurança do colaborador e entrega eficaz e efetiva do produto final para o cliente [14].

Portanto, a proposta de melhoria de processo por paradas de linha de produção, bem como a diminuição desse tempo à luz do que ocorre em toda a cadeia de produção de uma empresa do setor automotivo, permite a implementação da mineração dos dados coletados pelos sistemas de controle do processo de manufatura, a fim de que sejam encontrados padrões de perdas produtivas, com proposta através da análise realizada de diminuir os impactos na produção, em conjunto com o uso de ferramentas empíricas de gestão de processos, como o W.C.M.

\subsection{Mineração de Dados}

Mineração de Dados (Data Mining, em tradução livre) é o nome dado a um conjunto de técnicas e procedimentos que tenta extrair informações de nível semântico mais alto a partir de dados brutos, em outras palavras, permitindo a análise de grandes volumes de dados para extração de conhecimento [1], [3].

Este conhecimento pode ser na forma de regras descritivas dos dados, modelos que permitem a classificação de dados desconhecidos a partir de análise de dados já conhecidos, previsões, detecção de anomalias, visualização anotada ou dirigida, entre outros. Embora muitas destas abordagens tratem com dados tabulares, é possível extrair informações 52 tabulares de dados estruturados de forma diferente, como na Web. [11].

Para identificarmos quando uma base de dados é adequada para uma mineração de dados ou não, o conjunto de dados deve representar, de forma confiável, o universo (mundo real) a ser investigado, possibilitando assim inferir a situação problema como um todo, seja pela perspectiva de completeza ou complexidade do problema. Um dos "mitos" criados, a partir da motivação inicial das discussões sobre a mineração de dados, era ser uma alternativa para "grandes" bases de dados [4], [9].

Este fato decorreu da própria dificuldade de processamento inerente à descoberta e identificação de informações oportunas ao processo decisório em grandes conjuntos.

Como muitas bases de dados sofrem interferências de diversas formas, a elas atribuimos o nome de ruído. "Ruído" representa conteúdo nas bases de dados que pode prejudicar a qualidade da informação extraída, a partir de qualquer método, seja ele tradicional ou baseado em estratégias mais elaboradas. Destacam-se como ruídos: valores fora do domínio, ausência de valores, inconsistências, dentre outras [9], [11].

É importante lembrar que o mundo real é ruidoso, ou seja, se uma base de dados representa uma abstração deste mundo real, esta será ruidosa a despeito dos esforços despendidos para a sua modelagem e respectiva população. Cabe aos profissionais da área de tecnologia da informação minimizar o impacto negativo que estes ruídos possam representar nas informações extraídas e disponibilizadas aos gestores. Por exemplo, todas as vezes que, ao informar os dados cadastrais se omite ou não se informa corretamente a renda, gera-se um ruído no conjunto de dados [11].

\subsection{Trabalhos Relacionados}

Processos de Manufatura Inteligente demandam aplicações de alta complexidade, no que se refere a estudos de caso que indiquem soluções para, por exemplo, problemas de falta de qualidade no item manufaturado, ou até mesmo paradas de produção provocadas por falha em equipamentos. A integração de sistemas de automação com soluções de Internet das Coisas (do inglês Internet of Things, com sigla IoT) permite a aquisição de grande volume de informações, o que muitas vezes deixam de ser analisados por falta de conhecimento para tal [15]. 
Para esses e outros casos ligados a otimização da produtividade em indústria e processos de manufatura, a mineração de dados surgiu com o propósito de extração de informações, que eram desconhecidas até então, e assim torná-las válidas, bem como compreensíveis, de grandes bases de dados com o intuito de melhorar e otimizar as tomadas de decisão que devem ser feitas pelo gerente do negócio [9].

Técnicas diversas para extração de informação de grande quantidade de dados, tais como Random Forest, redes Bayseanas, algoritmos de classificação, redes neurais artificiais (da sigla RNA), árvores de decisão, sistemas de lógica Fuzzy e algoritmos genéticos, são utilizadas como propostas de solução, dependendo do cenário que exige solução de problema proposto [4], [6]-[8], [16].

Em se tratando da abordagem Random Forest, este método consiste nas correlações que são estimadas de acordo com os resultados de saída de várias árvores de decisão, onde esses diversos resultados das saídas são combinados, para assim termos uma saída única. Cada resultado de saída corresponde a uma "escolha" que é feita pela árvore de decisão, em sua unidade, sendo que cada árvore trabalha com um trecho de amostras da base de dados, onde esses trechos são aleatórios e de mesmo tamanho, para que não haja desbalanceamento das sub-bases. A combinação de todas as escolhas denota na escolha global, onde cada árvore de decisão que participa do processo possui igual poder de decisão no valor de saída final, sendo esse do Random Forest, correspondente então a base de dados completa [17].

Aplicações do Random Forest para detecção de defeitos em soldas feitas por robô [17], bem como detecção de falhas em camadas de dispositivos semicondutores em sua fase de fabricação [16] são exemplos de situações onde o Random Forest foi aplicado para previsão de situações que estavam fora do padrão esperado, para controle do processo de manufatura avançada e de qualidade exigido em cada cenário citado.

\section{Materiais e Métodos}

\subsection{Descrição da base de dados}

A Base de dados analisada possui, em tamanho total, 1.2GB de informações, contendo na mesma 12 colunas com 579.237 linhas de informações acerca dos mais variados tipos de operações que resultaram em paradas de linha. Foram levantados dados de 2018 até 2020 de histórico de paradas de linha, com extração realizada em um banco de dados SQL. A Tabela 1 mostra dicionário de dados montado.

Tabela 1: Dicionário de dados utilizado para previsão

\begin{tabular}{l|l|l}
\hline Nome do campo & $\begin{array}{l}\text { Tipo } \\
\text { de } \\
\text { dado }\end{array}$ & Descrição \\
\hline ProductionOrderId & Long & Número de batismo do veículo. \\
\hline CallStartTime & Time & $\begin{array}{l}\text { Tempo que marca o início do } \\
\text { alerta de da parada de linha. }\end{array}$ \\
\hline Time_DIFF & Time & $\begin{array}{l}\text { Tempo que marca o fim de } \\
\text { parada de linha. }\end{array}$ \\
\hline StopLineStartTime & Time & $\begin{array}{l}\text { Tempo que marca o início de } \\
\text { parada de linha. }\end{array}$ \\
\hline Ciferença de tempo entre \\
\hline CallEndTime - CallStartTime
\end{tabular}

\subsection{Análise Descritiva dos Dados}

Temos, de parte dos atributos numéricos, os tipos de dados sinalizados como Time, que correspondem a todos os tempos de início e fim de parada de linha na produção. Já os que estão indicados como sendo de atributo nominal, que são do tipo String, representam o local onde houve a parada de linha, o modo de parada - se parada executada por ação humana, ou máquina - e qual tipo de operação foi impactada com a parada de linha.

DOI: 10.25286/repa.v6i3.1687 
Em relação a este último - nome do campo Operation - temos a classificação em: parada por conta de ferramentas (índice SCR, GEN, F), paradas por falta de qualidade na operação (tipos QCE), parada por falta de finalização do processo (tipos PCE) e paradas por falta de leitura de peças montadas (TRA, TRP, OGG, CERT, CEL). O tipo de dado que ficou sinalizado como Long representa o número de batismo da carroceria, o mesmo que vai na documentação do carro e fica atrelado ao chassi do mesmo. Toda vez que existe uma parada de linha na produção, o valor de início, fim, qual estação de trabalho e qual operação sofreu impacto é registrado no sistema de manufatura.

Através da tabela de informações sobre as paradas de linha, foram escolhidos como atributos numéricos a diferença de tempo entre o início e fim da parada (chamado de CallstartTime e CallIEndTime, respectivamente) e como atributo de classe se a parada de linha foi gerada por operador (nomeada de Operator), ou por falha no processo (Nomeada de Fail).

Extrações de gráficos também foram realizadas, de tal forma que foram expostos na primeira análise de tempo, todos os valores de tempo encontrados, em segundos, conforme Figura 1.

Diante de melhor análise a se tornar evidente, foi realizada nova extração mostrada na Figura 2, essa com os primeiros 1000 segundos de paradas, pois foi percebido que as paradas de linha estavam mais concentradas nesse intervalo de tempo.

Logo em seguida, para refinamento ainda mais detalhado da informação, foram evidenciados os 50 primeiros valores medidos de tempo, também em segundos, da quantidade de ocorrências de parada de linha de produção (ou seja, quantas vezes uma parada de 1 segundo influenciou no total de paradas), exibidos na Figura 3.

\subsection{Pré-processamento dos dados}

Por critério de seleção, foram escolhidas as colunas CallStartTime e CallEndTime para análise a diferença de tempo de parada de linha na produção, e assim criada uma nova coluna chamada Time_Diff. Também foi realizada a criação de uma coluna intitulada Type_BIN, que corresponde a informação binária da coluna Type, onde o índice 0 corresponde a todas as paradas relacionadas a operator e o índice 1 a todas as paradas relacionadas a fail das operações, constando sempre o tipo de operação que provocou a parada, na coluna Operation.

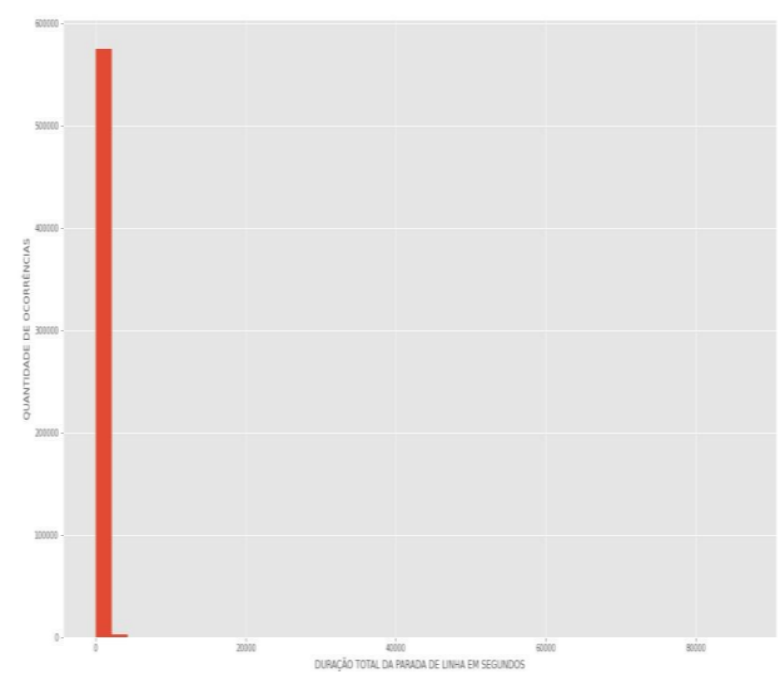

Figura 2: Análise do total de ocorrências de paradas de linha, em segundos.

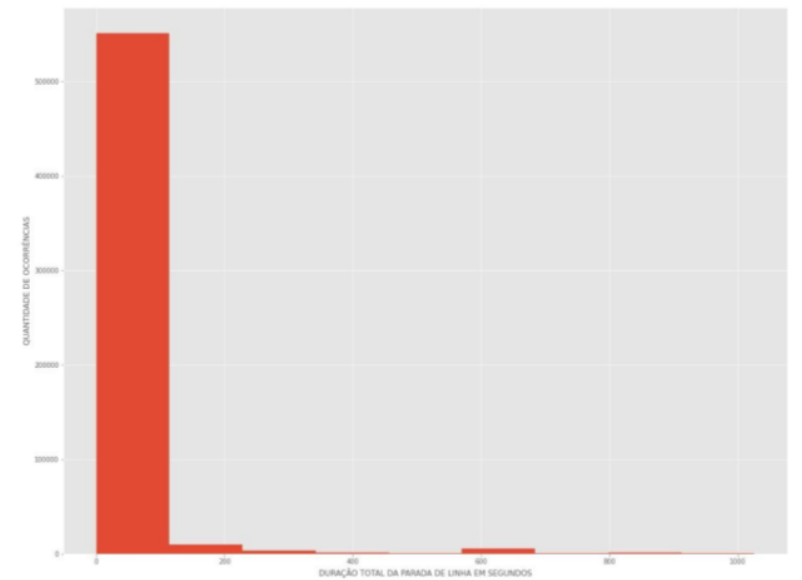

Figura 3: Análise dos primeiros 1000 segundos de parada de linha.

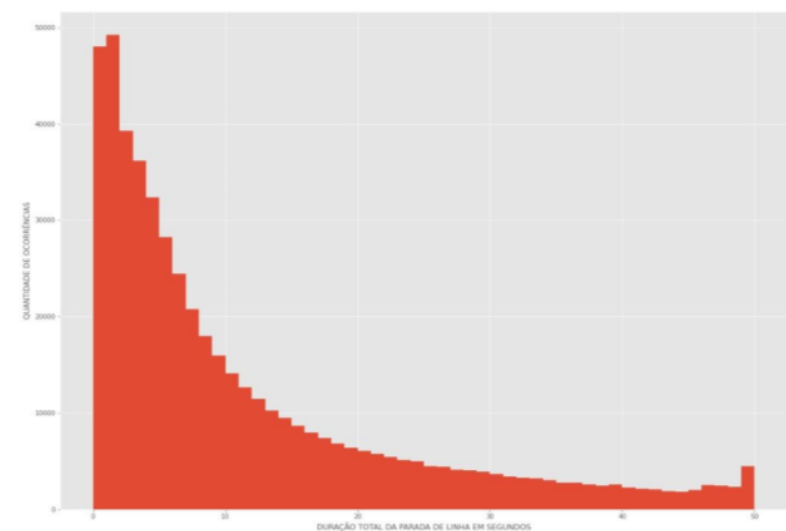

Figura 4: Análise dos 50 primeiros valores de tempo, em segundos, correspondente as ocorrências de paradas de linha. 
Para também melhorar a execução de nosso algoritmo, como também termos uma categorização das classes de maneira mais adequada, foram criadas novas colunas, em formato de labels, das colunas trabalhadas nesta base de dados, dos quais:

- Workplace_Category: label encoder de workplace;

- Operation_Category: label encoder de operation;

- $\quad$ Criação de StoplineShift (período do dia da parada): com label enconder, categorizada para StopLineShift_Category, se no primeiro, segundo ou terceiro turno de produção;

- $\quad$ Criação de StopLineCriticality (criticidade da parada): com label encoder, categorizada para StopLineCriticality_Category, criticidade da parada de linha;

- Chassis_Category - label enconder de ProductionOrderId.

Também foram necessárias algumas transformações de colunas e variáveis, para correto ajuste da base de dados disponibilizada ao modelo de previsão, que foram as seguintes:

- TimeDiffSeconds: transformação de TIME_DIFF em segundos;

- Criação da coluna Time_diff_seconds_bins: distribuição da classe TimeDiffSeconds em ranges de tempo, para melhor análise da abordagem de Random Forest na base de dados;

- $\quad$ Colunas da base de dados utilizadas para treinamento do modelo: ["Chassis_Category", "Type_BIN", "Workplace_Category", "Operation_Category", "Time_diff_seconds_bins", "StopLineShift_Category", "StopLineCriticality_Category"]

Finalmente, temos a organização tanto da base de dados, como dos gráficos gerados e do treinamento e validação do modelo no formato de arquivos de processamento, debug e geração de dados no formato de plataforma colaborativa, como exemplo a que foi utilizada, o Google Colab. $\mathrm{Na}$ plataforma em questão, foram elencados coluna para as seguintes distribuições:

- Turno de ocorrência da parada de linha, se primeiro, segundo ou terceiro turno de produção;
- $\quad$ Criticidade da parada de linha, de acordo com a quantidade, em segundos da parada de linha. Os indicadores para criação dessa nova coluna foram sinalizados como:

- Se tempo de parada entre 0 e 540 segundos, criticidade baixa;

○ Se tempo de parada entre 541 e 1140 segundos, criticidade média;

- Se tempo de parada entre 1141 e 1740 segundos, criticidade alta;

- Se tempo de parada entre 1741 e 2340 segundos, criticidade altíssima;

- Se tempo de parada maior que 2341 segundos, criticidade desastre.

\subsection{Metodologia Experimental}

Primeiramente, a coleta de informações foi realizada através de um arquivo .csv disponibilizado pelo stakeholder, para fins de análise e tratamento das linhas, quando necessário, com características já citadas em 3.1. Logo após, foram definidas quais transformações seriam necessárias para adaptação da base de dados, e assim ter a possibilidade de manipulação o mais adequada possível dos dados, tanto para a aquisição de gráficos relativos a paradas de linha de produção, e sua quantidade de paradas de acordo com a estação de trabalho, por exemplo, como para o modelo de previsão proposto, para nosso cenário o Random Forest, conforme mencionado em 3.3.

Em seguida, foi realizado o processamento da base, de acordo com os atributos de classe escolhidos e assim categorizados, para melhor análise e performance do modelo utilizado. Com isso, foi possível a extração total da quantidade de paradas de linha existentes, do tempo que cada uma dessas paradas de linha influenciou na linha de produção, a tendência de maior ocorrência de paradas de linha, por exemplo, se quando com tempo menor que 60 segundos ou maior que 60 segundos.

Finalmente, já para o modelo de previsão utilizado, as colunas criadas em 3.3 suportaram a estimativa dos valores encontrados, tanto para a parte da base de dados que foi utilizada para treinamento do modelo, como para validação do mesmo. Foi feita uma distribuição de classes, onde os períodos de tempo que houveram maiores 
ocorrências de parada de linha recebem o menor intervalo de classes, para assim a previsão ocorrer o mais próxima do esperado, em se tratando da realidade de paradas de linha da produção, e um balanceamento da quantidade de amostras de cada classe, de tal forma que para a base de treinamento ter a mesma quantidade de amostras da classe com menor quantidade de amostras, que no caso foi a classe 10 com 2410 amostras, independente do intervalo de classes, fazer o treinamento da previsão o mais balanceado possível.

Adicionalmente, das classes com mais amostras foi realizado uma permuta de índices, para validação do treinamento. Com o proposto, conseguimos uma estratificação de 12 intervalos de classe, que nos permite assim identificar melhor os padrões de criticidade de parada de linha. Os intervalos das classes estão apresentados na Figura 5:

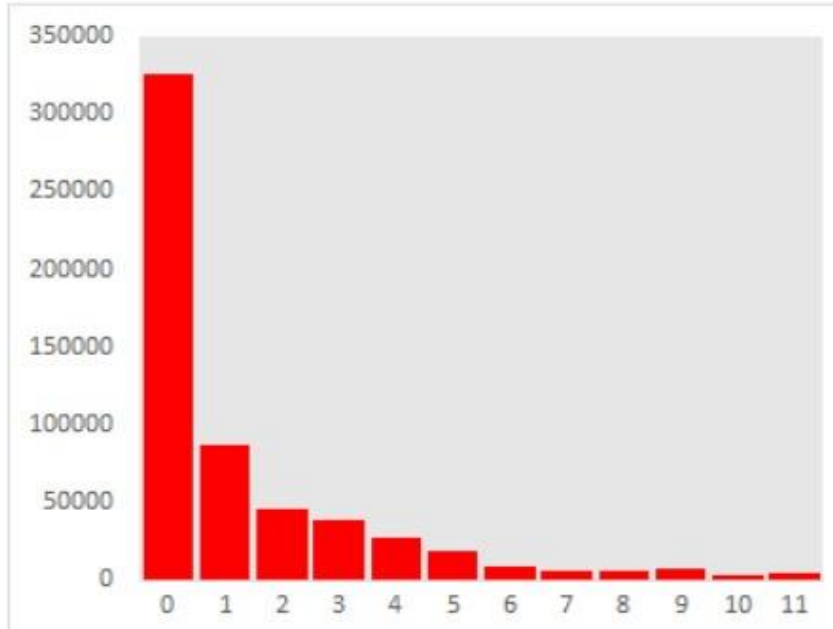

Figura 5: Gráfico contendo a estratificação da quantidade de amostras, de acordo com as classes separadas para previsão das paradas de linha.

\section{Resultados}

Antes do balanceamento das amostras nas classes ser realizado, conforme comentado em 3.4, o modelo de previsão fez o processamento das informações contidas na base de dados, de acordo com a quantidade de amostras presentes em cada intervalo de classes, desconsiderando assim o mínimo valor de amostras apontado na classe 10 utilizado para, posteriormente, balancear a quantidade de amostras nas demais classes, de 2410 registros. Eis que os resultados obtidos pela precisão do modelo Random Forest seguem abaixo:
Tabela 2: Valores de acurácia do modelo de previsão, sem o balanceamento da quantidade de amostras entre as classes.

$\begin{array}{rrrrr} & \text { precision } & \text { recall } & \text { f1-score } & \text { support } \\ 0 & 0.75 & 0.71 & 0.73 & 68834 \\ 1 & 0.19 & 0.21 & 0.20 & 16129 \\ 2 & 0.13 & 0.14 & 0.14 & 8374 \\ 3 & 0.14 & 0.15 & 0.14 & 7173 \\ 4 & 0.21 & 0.23 & 0.22 & 5074 \\ 5 & 0.11 & 0.12 & 0.12 & 3599 \\ 6 & 0.09 & 0.10 & 0.09 & 1432 \\ 7 & 0.08 & 0.10 & 0.09 & 1147 \\ 8 & 0.20 & 0.20 & 0.20 & 1192 \\ 9 & 0.68 & 0.67 & 0.67 & 1585 \\ 10 & 0.45 & 0.48 & 0.46 & 439 \\ 11 & 0.98 & 0.99 & 0.98 & 870 \\ \text { accuracy } & & & & \\ \text { macro avg } & 0.33 & 0.34 & 0.51 & 115848 \\ \text { weighted avg } & 0.52 & 0.51 & 0.52 & 115848 \\ & & & & \end{array}$

Com a acurácia em $51 \%$, foi necessário reanalisar o pré-processamento, para entendimento dos valores, e foi constatado que seria necessário uma nova distribuição de amostras entre as classes era necessária para uma nova validação do modelo de previsão, mantendo como meta os intervalos de tempo que possuem maiores ocorrências de parada de linha, como o menor intervalo intra-classe.

Sendo assim, a validação do modelo fica mais detalhada, de tal forma a saber se várias paradas de criticidade baixa influenciam com maior ou menor frequência do que uma parada longa que causa um valor alto de parada de linha de produção. Depois do balanceamento das classes, temos o seguinte resultado para a previsão:

Tabela 3: Valores de acurácia do modelo de previsão, com o balanceamento da quantidade de amostras entre as classes, baseado na classe com menor quantidade de índices, no caso a classe 11, com 2410 registros.

$\begin{array}{rrrrr} & \text { precision } & \text { recall } & \text { f1-score } & \text { support } \\ 0 & 0.40 & 0.32 & 0.35 & 300 \\ 1 & 0.18 & 0.17 & 0.18 & 260 \\ 2 & 0.15 & 0.18 & 0.17 & 204 \\ 3 & 0.19 & 0.19 & 0.19 & 241 \\ 4 & 0.25 & 0.24 & 0.25 & 246 \\ 5 & 0.15 & 0.16 & 0.15 & 215 \\ 6 & 0.16 & 0.17 & 0.17 & 225 \\ 7 & 0.24 & 0.23 & 0.23 & 245 \\ 8 & 0.35 & 0.33 & 0.34 & 256 \\ 9 & 0.51 & 0.56 & 0.53 & 223 \\ 10 & 0.71 & 0.72 & 0.71 & 237 \\ 11 & 0.99 & 0.99 & 0.99 & 240 \\ & & & & \\ \text { accuracy } & & & 0.36 & 2892 \\ \text { macro avg } & 0.36 & 0.36 & 0.35 & 2892 \\ \text { weighted avg } & 0.36 & 0.36 & 0.36 & 2892\end{array}$


Tabela 4: Valores de acurácia do modelo de previsão, com o balanceamento da quantidade de amostras entre as classes, baseado na classe com menor quantidade de índices, no caso a classe 11, com 2410 registros, variando em termos de amostras, conforme as já utilizadas em primeira validação do modelo.

$\begin{array}{rrrrr} & \text { precision } & \text { recall } & \text { f1-score } & \text { support } \\ 0 & 0.51 & 0.31 & 0.39 & 1173 \\ 1 & 0.21 & 0.20 & 0.20 & 781 \\ 2 & 0.14 & 0.16 & 0.15 & 629 \\ 3 & 0.17 & 0.19 & 0.18 & 636 \\ 4 & 0.26 & 0.28 & 0.27 & 668 \\ 5 & 0.13 & 0.17 & 0.15 & 544 \\ 6 & 0.17 & 0.20 & 0.19 & 603 \\ 7 & 0.18 & 0.20 & 0.19 & 656 \\ 8 & 0.41 & 0.31 & 0.35 & 941 \\ 9 & 0.49 & 0.71 & 0.58 & 503 \\ 10 & 0.82 & 0.71 & 0.76 & 838 \\ 11 & 0.97 & 0.99 & 0.98 & 753 \\ & & & & \\ c y & & & 0.38 & 8725 \\ \text { vg } & 0.37 & 0.37 & 0.37 & 8725 \\ \text { vg } & 0.40 & 0.38 & 0.38 & 8725\end{array}$

A avaliação que é feita também para treinamento do modelo, considerou a substituição de valores onde tivemos classes com mais do que 2410 registros, e não houve mudança brusca nos valores de precisão atingidos, conforme tabelas 5 e 6 acima, que registraram $36 \%$ e $38 \%$ de previsão final, respectivamente. Houve considerável melhora nas previsões das classes 5 até a 11, que para o gerente do negócio torna-se mais interessante saber, por exemplo, com mais precisão quando paradas registradas na base de dados que são acima de 1 minuto podem impactar o processo de produção.

Em se tratando das paradas com menos de 1 minuto, por mais que ocorram com maior frequência, é de se saber que são paradas que ocorrem, pelo presente estudo, por falta de adequação do tempociclo de operação dos colaboradores nas estações de trabalho.

\subsection{Discussão}

Com os resultados encontrados, o gerente do negócio da indústria estudada pode, então, ter tomadas de decisão de tal forma que as paradas de linha que mais impactam o processo são aquelas em que:

- O templo-ciclo de execução da operação não está conforme o previsto para o ritmo da linha de produção, ou seja, curto espaço de tempo para várias atividades na estação de trabalho serem exercidas;

- $\quad$ Falta ou reciclagem de treinamento e capacitação do colaborador em realizar as atividades, de acordo com a estação de trabalho;

- $\quad$ O número de carros por hora que são fabricados na linha não está de acordo com a quantidade de colaboradores exigidos para execução da quantidade de atividades nas estações de trabalho;

- Quebras ocasionadas por ferramentas do processo precisam de maior monitoramento do time da Manutenção, com relação a ações de execução das atividades necessárias de manutenção preventiva/preditiva.

\section{Conclusões e Trabalhos Futuros}

O perfil de perda a ser previsto auxiliará na tomada de decisão do gerente do negócio quando, por exemplo, eventos de paradas de programação para reparação dos equipamentos serão necessários de acontecer, para prevenir grandes paradas de linha.

É esperado, também, com os valores encontrados pela previsão, aumento na eficiência e eficácia em cada estação de trabalho, considerando que correções no tempo-ciclo de operação já citados sejam realizados, para assim atingir o objetivo da previsão mais próxima da realidade de modos de falha para as paradas de linha que ocorrem na manufatura.

Como proposta de trabalho futuro, a correlação dessa base de dados com outras bases de dados que coletam informações distintas de perdas produtivas, como perdas por atraso logístico externa a cadeia de produção, perdas por falha de outras oficinas ligadas a entrega de peças e carrocerias para manufatura, a saber da oficina de Prensas de chapas e do parque de fornecedores.

Finalmente, também segue a proposta da aplicação de outras técnicas de previsão, a saber séries temporais, algoritmos genéticos, e quaisquer outras técnicas de algoritmos de aprendizado de máquina ou de inteligência artificial, que possa retornar valores adequados para auxílio do gerente do negócio na tomada de decisão. 


\section{Referências}

[1] M. G. B, S. Chren, B. Rossi, and T. Pitner, for Smart Grid Systems, vol. 1. Springer International Publishing, 2019.

[2] M. Syafrudin, G. Alfian, N. L. Fitriyani, and J. Rhee, "Performance analysis of IoT-based sensor, big data processing, and machine learning model for real-time monitoring system in automotive manufacturing," Sensors (Switzerland), vol. 18, no. 9, 2018.

[3] L. Rokach and O. Maimon, "Data mining for improving the quality of manufacturing: A feature set decomposition approach," J. Intell. Manuf., vol. 17, no. 3, pp. 285-299, 2006.

[4] C. Gröger, F. Niedermann, and B. Mitschang, "Data mining-driven manufacturing process optimization," Lect. Notes Eng. Comput. Sci., vol. 3, pp. 1475-1481, 2012.

[5] G. Filios, "An Agnostic Data-Driven Approach to Predict Stoppages of Industrial Packing Machine in Near Future," pp. 236-243, 2020.

[6] C. Dudas, M. Frantzén, and A. H. C. Ng, "A synergy of multi-objective optimization and data mining for the analysis of a flexible flow shop," Robot. Comput. Integr. Manuf., vol. 27, no. 4, pp. 687-695, 2011.

[7] C. Dudas, A. H. C. Ng, L. Pehrsson, and H. Boström, "Integration of data mining and multiobjective optimisation for decision support in production systems development," Int. J. Comput. Integr. Manuf., vol. 27, no. 9, pp. 824-839, 2014.

[8] A. Luckow et al., "Artificial Intelligence and Deep Learning Applications for Automotive Manufacturing," Proc. - 2018 IEEE Int. Conf. Big Data, Big Data 2018, pp. 3144-3152, 2019.

[9] K. Wang, "Applying data mining to manufacturing: The nature and implications," $\mathrm{J}$. Intell. Manuf., vol. 18, no. 4, pp. 487-495, 2007.

[10] A. Majeed, J. Lv, and T. Peng, "A framework for big data driven process analysis and optimization for additive manufacturing," 58
Rapid Prototyp. J., vol. 25, no. 2, pp. 308-321, 2019.

[11] P. C. Ncr et al., "Step-by-step data mining guide," SPSS inc, vol. 78, pp. 1-78, 2000.

[12] C. J. Muller, "A evolução dos sistemas de manufatura e a necessidade de mudança nos sistemas de controle e custeio," p. 222, 1996.

[13] L. C. Maia, a. C. Alves, and C. L. Leão, "Metodologias Para Implementar Lean Production: Uma Revisão Critica De Literatura," CILME'2011, p. 0915A, 2011.

[14] F. Pereira, L. Carelli, C. Manuel, T. Rodriguez, and L. M. Rôa, "Proposta de adequação do processo de inspeção com base nos conceitos do lean manufacturing : estudo de caso em um fabricante de equipamentos agrícolas.," vol. 1, p. 86, 2016.

[15] D. Wu, C. Jennings, J. Terpenny, R. X. Gao, and S. Kumara, "A Comparative Study on Machine Learning Algorithms for Smart Manufacturing: Tool Wear Prediction Using Random Forests," J. Manuf. Sci. Eng. Trans. $A S M E$, vol. 139, no. 7, 2017.

[16] L. Puggini, J. Doyle, and S. McLoone, "Fault detection using random forest similarity distance," IFAC-PapersOnLine, vol. 28, no. 21, pp. 583-588, 2015.

[17] Z. Zhang, Z. Yang, W. Ren, and G. Wen, "Random forest-based real-time defect detection of Al alloy in robotic arc welding using optical spectrum," J. Manuf. Process., vol. 42, no. April, pp. 51-59, 2019. 